\title{
多电荷环糊精的超分子组装
}

\author{
张 依刘 育* \\ (南开大学化学系 元素有机化学国家重点实验室 天津 300071)
}

\begin{abstract}
摘要 多电荷环糊精作为一类具有优异的水溶性、低生物毒性和高电荷密度的大环主体，可与有机、无机和生物分子 通过疏水的空腔和静电相互作用等多种协同作用力构筑具有稳定拓扑形貌、功能多样性和刺激响应性的智能超分子组 装体而被广泛研究. 综述了近年来以正/负电荷及两亲性多电荷环糊精作为主体构筑的具有 $\mathrm{pH}$ 、光、酶、氧化还原、 磁和多重刺激响应的智能超分子组装体以及其在药物传递、控制释放和传感检测等领域应用的最新研究进展, 并对多 电荷环糊精智能超分子组装体所面临的挑战和未来的发展进行了探讨.
\end{abstract}

关键词 多电荷环糊精; 超分子组装体; 刺激响应性; 智能材料

\section{Supramolecular Assemblies of Multi-Charged Cyclodextrins}

\author{
Zhang, Yi Liu, Yu*
}

(State Key Laboratory of Elemento-organic Chemistry, College of Chemistry, Nankai University, Tianjin 300071)

\begin{abstract}
As a class of macrocyclic host with excellent water solubility, low biotoxicity and high charge density, multicharged cyclodextrins have been extensively studied because they can interact with organic/inorganic/biological molecules through multiple synergistic forces such as the hydrophobic cavities and electrostatic interactions to construct the smart supramolecular assemblies with stable topology, functional diversity and stimulus responsiveness. The latest research progress of pH-, photo-, enzyme-, redox-, magnetic- and multi-stimulus responsive smart supramolecular assemblies, which are constructed by typical positively/negatively charged and amphiphilic multi-charged cyclodextrins including their application in the fields of drug delivery, controlled release and sensory detection is introduced, and the challenges and future developments of multi-charged cyclodextrin smart supramolecular assemblies are discussed.

Keywords multi-charged cyclodextrin; supramolecular assembly; stimulus responsiveness; smart material
\end{abstract}

环糊精(cyclodextrin, 简称 $\mathrm{CD}$ ) 作为超分子化学研 究的第二代大环主体化合物, 自从被发现以来就受到科 研工作者的持续关注. 其本质是由 $D$-吡喃葡萄糖通过 $\alpha-1,4-$ 糖苷键环化而成的环状低聚糖, 具有截雉状拓扑 结构和 “内外” 两亲的特性. 其中, 常见的 $\alpha 、 \beta 、 \gamma$-环 糊精分别具有 $6 、 7 、 8$ 个吡喃葡萄糖单元. 环糊精因其 具有良好的水溶性、生物相容性以及廉价易得等特点被 广泛应用于日用化工、农业、催化、智能材料和生物医 学等领域 ${ }^{[1]}$. 然而, 较低的溶解度和有限的包结能力限 制了天然环糊精的进一步应用. 为了进一步提高分子的 选择性和键合能力以及功能的多样性, 许多环糊精衍生 物被设计和合成出来. 众所周知, 环糊精大口端和小口 端拥有的众多羟基可被多种功能基团修饰, 功能基团的
类型、数目和修饰位置可以配合其空腔衍生出具有不同 性能的大环主体. 其中, 多电荷环糊精是一类带有多个 正电荷或者负电荷基团的典型大环主体分子，具有诸多 优点: (1)带电基团的引入大大改善了环糊精的水溶性和

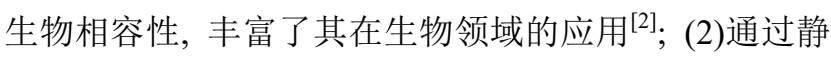
电相互作用力能够显著地提高带相反电荷客体分子的 识别能力, 增强分子选择性 ${ }^{[3]}$; (3)修饰后的环糊精衍生 物空腔变深, $\mathrm{CD}$ 部分或者取代基部分与客体之间的相 互作用增强，可以实现超分子纳米组装体的高稳定性和 紧密性 ${ }^{[4]}$. 更重要的是多电荷环糊精的高电荷密度使其 成为基于分子诱导聚集策略构筑超分子组装体的最理 想主体之一。

智能体系是一类当受到外部信号刺激时，可以给出

\footnotetext{
* Corresponding author. E-mail: yuliu@nankai.edu.cn

Received April 25, 2020; revised April 29, 2020; published online May 11, 2020.

Dedicated to the 40th anniversary of Chinese Journal of Organic Chemistry

Project supported by the National Natural Science Foundation of China (Nos. 21772099, 21861132001).

国家自然科学基金(Nos. 21772099, 21861132001)资助项目.
} 
特定反馈的新型功能体系, 具体的反馈主要来源于体系 内部结构的变化. 刺激信号也可能是多种多样的, 例如 $\mathrm{pH}$ 、氧化还原、酶、温度、光、磁力、超声和电压等. 环 糊精与客体分子的结合主要利用具有动态和可逆性质 的非共价键作用力，提供了一种有效的方式来模仿自然 和生理的响应过程. 通过在环糊精超分子体系中引入特 定的响应基团, 合适地调整外部条件, 将基于 $\mathrm{CD}$ 的纳 米体系结构破坏、重组、甚至降级为原始的构建模块, 这 样的超分子体系具备智能材料的性能 ${ }^{[5]}$. 基于多电荷环 糊精的智能组装体对外部刺激的响应使主客体组装模 式发生变化主要来源于三个方面: (1)主/客体分子上电 荷数量或者电性的改变使主客体之间的静电相互作用 受影响从而改变组装模式; (2)客体分子性质的变化使其 从环糊精的空腔中逸出, 影响了主客体相互作用, 破坏 了组装模式; (3)主/客体分子的降解或功能基团的离去 导致组装体的解体. 近些年来, 科研工作者利用非共价 键自组装技术更快、更方便、更灵活地构建了许多结构 新颖的功能化超分子组装体, 在纳米超分子领域取得一 系列创新性突破, 极大地推进了超分子体系在智能识别 与组装、新型生物医药功能材料等领域的应用.

多电荷环糊精智能组装体的设计理念是将智能化 学方法赋予缺乏环境响应特性的离子大环受体体系中, 也就是说将刺激反应位点以共价或非共价方式引入 $\mathrm{CD}$. 因此, 构筑的多电荷环糊精超分子组装体具有 $\mathrm{pH}$ 、光、 酶、氧化还原和磁等多种刺激响应性, 从而提高组装体 的生物相容性和多功能性, 拓展超分子的应用领域. 本 综述将从典型的正/负电荷及两亲性多电荷环糊精构筑 的超分子组装体出发, 根据组装体常见的刺激响应类型 介绍多电荷环糊精智能超分子体系的最新进展; 同时, 关注了组装体结构和响应的对应关系以及其在药物传 递、控制释放和传感检测领域的应用.

\section{1 基于多电荷环糊精的超分子组装}

由于环糊精的多电荷衍生化赋予环糊精主体更加 丰富的性质, 科研工作者会根据不同的研究目的来选择 合适的带电基团, 并修饰到环糊精上以获得具有有序结 构和独特功能的组装体. 在以下部分, 根据组装体主体 分子的不同, 选取典型的实例进行简要的讨论与介绍.

\section{1 负电荷环糊精}

典型的负电荷环糊精是以羧基、磺酸基等作为修饰 基团. 相比天然环糊精, 这类环糊精负电荷密度比较高, 在分子识别和分子组装方面对正电荷的客体分子具有 优异的识别能力和较好诱导聚集能力, 可以通过非共价 相互作用构筑功能性超分子组装体.

负电荷基团的修饰增强了环糊精与药物分子的相
互作用，早些年，Zhang 等 ${ }^{[6]}$ 发现羧基取代的 $\gamma-\mathrm{CD}$ 可以 通过静电相互作用实现对神经肌肉阻断剂罗库溴铵选 择性键合，使该环糊精被广泛用于手术后去除罗库溴 铵. Wenz 等 ${ }^{[4 b]}$ 发现 6 位全磺化环糊精可以与抗癌药喜树 碱通过非共价相互作用构筑成稳定的包合物，大大增加 了喜树碱的水溶性. 这些研究为负电荷环糊精在生物医 药领域的应用奠定了基础. 此外, 在最近的研究中, Tapparel 团队 ${ }^{[7]}$ 发现磺化环糊精可以作为广谱抗病毒试 剂. 如图 1, 作者合成了三种类型的磺化环糊精 CD1、 $\mathrm{CD} 2$ 和 $\mathrm{CD} 3$ 来模拟乙酰肝素硫酸盐用于抗病毒. 实验 结果表明, 这三种磺化环糊精均具有广谱抗病毒活性, 并揭示了柔性链和刚性链对抗病毒能力的影响. 这个工 作拓展了多电荷环糊精在抗病毒药物研发领域的应用.

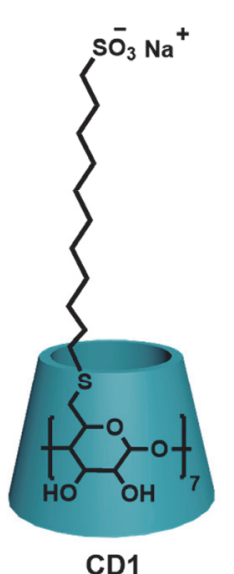

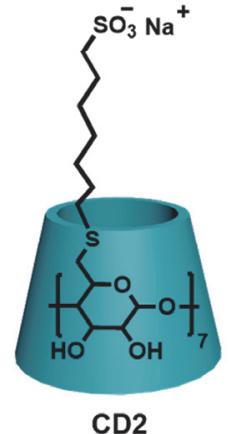

CD2

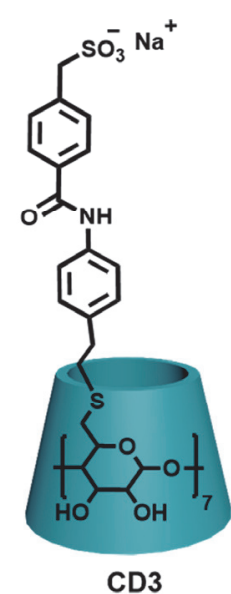

CD3
图 1 三种磺化环糊精的结构 ${ }^{[7]}$

Figure 1 Structures of three sulfonated $\mathrm{CDs}^{[7]}$

静电作用的引入使负电荷环糊精在构筑功能性超 分子组装体中也表现出优异的性质. 我们课题组 ${ }^{[8]}$ 报道 了通过羧酸化环糊精(CCD)诱导含有季铵头基的正电荷 两亲客体分子聚集形成的超分子组装体. 作者合成了单 羧酸取代 $\beta$ - $\mathrm{CD}$ 、单羧酸取代全甲基化 $\beta$ - $\mathrm{CD}$ 和全羧酸取 代 $\beta$-CD 三种主体化合物, 研究了它们对正电荷客体的 聚集诱导行为. 其中, 只有全羧基取代 $\beta$ - $\mathrm{CD}$ 展现出良 好的诱导聚集能力, 突出了静电相互作用在分子诱导聚 集中的重要作用. 此外, 作者还利用这种组装机制实现 了对模型药物分子的负载与缓慢释放. 该组装体为新型 纳米胶囊的构筑提供了理论基础. 另一个典型例子是利 用磺化环糊精通过静电相互作用诱导低聚对苯撑乙烯 衍生物(OPV-I) 聚集形成纳米粒子，再将荧光染料尼罗 红(NiR)负载到 SCD/OPV-I 纳米组装体的疏水层中构筑 成新型高效的水相人工光捕获体系(图 2) ${ }^{[9]}$. 体系中 $\mathrm{SCD} / \mathrm{OPV}-\mathrm{I}$ 作为荧光给体, NiR 作为荧光受体, 利用超 分子组装策略有效地拉近了给体与受体之间的距离, 实 现了高效的能量转移和超高的天线效应. 利用该策略构 
筑的光捕获体系为人工模拟自然界的光合作用提供了 新思路.

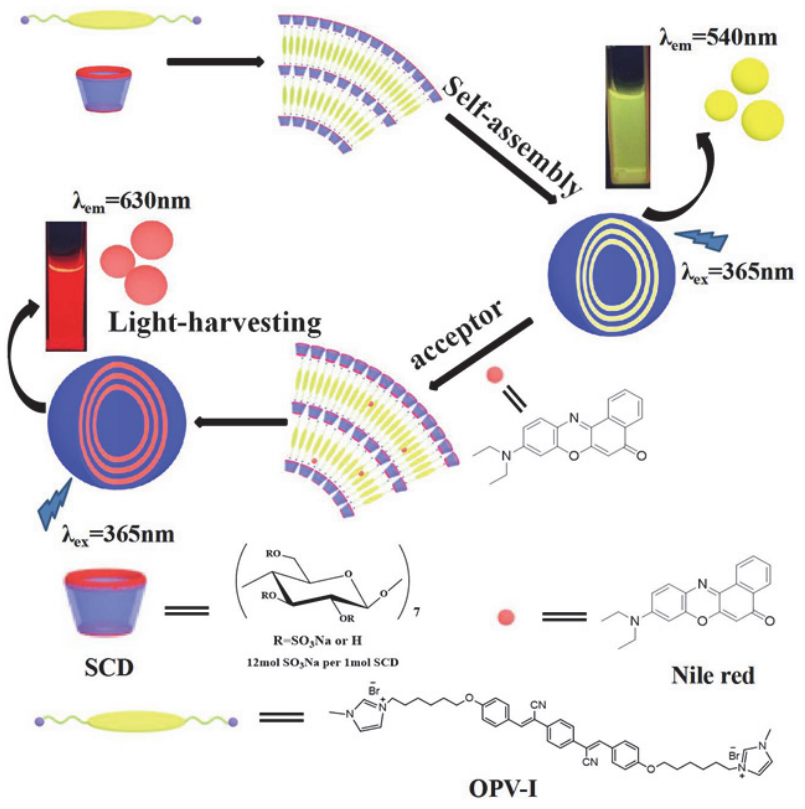

图 2 磺化环糊精构筑的光捕获体系 ${ }^{[9]}$

Figure 2 Light-harvesting system by sulfonated cyclodextrin ${ }^{[9]}$ Reproduced with permission. Copyright 2017, Wiley-VCH

\section{2 正电荷环糊精}

典型的正电荷环糊精是以氨基、胍基和咪唑基等作 为修饰基团. 正电荷基团的引入不仅增强了环糊精与负 电荷无机、有机客体的相互作用, 还促进其与生物大分 子 DNA 和 RNA 相互作用并形成纳米体系. 利用正电荷 环糊精构筑的功能性纳米组装体是超分子领域的研究 热点.

正电荷环糊精可以与负电荷表面活性剂相互作用 形成两亲超分子聚集体用作纳米载体 ${ }^{[10]}$. 另外, 还可以 构筑基因载体纳米体系, Fernández 等 ${ }^{[11]}$ 报道了氨基环 糊精适用于 DNA 传递, 实现了高转染效率和低毒性. 此外, 有研究将多氨环糊精通过二硫键修饰上叶酸, 利 用氨基环糊精对 DNA 的凝聚作用构筑成了靶向病变细 胞的基因转染体系 ${ }^{[12]}$. 如图 3 所示, 通过体系中叶酸的 靶向作用将 DNA 递送到特定肿瘤细胞内, 并利用细胞 内的谷胱甘肽切断二硫键释放叶酸, 使叶酸受体循环, 保证了叶酸受体介导的内吞作用的持续性, 从而实现高 效的基因表达. 该超分子基因递送体系有望用于癌症的 基因治疗.

正电荷环糊精不仅可以与生物分子作用用于生物 医药方面, 还可以与无机物相互作用构筑有机-无机杂 化体系. 如图 4 所示, 利用全氨基取代的环糊精 $(\mathrm{EDA}-\mathrm{CD})$ 与磷铇酸 $(\mathrm{POM})$ 相互作用构筑了超分子杂化 组装体并用于污染物的光降解 ${ }^{[13]}$. 研究结果显示在

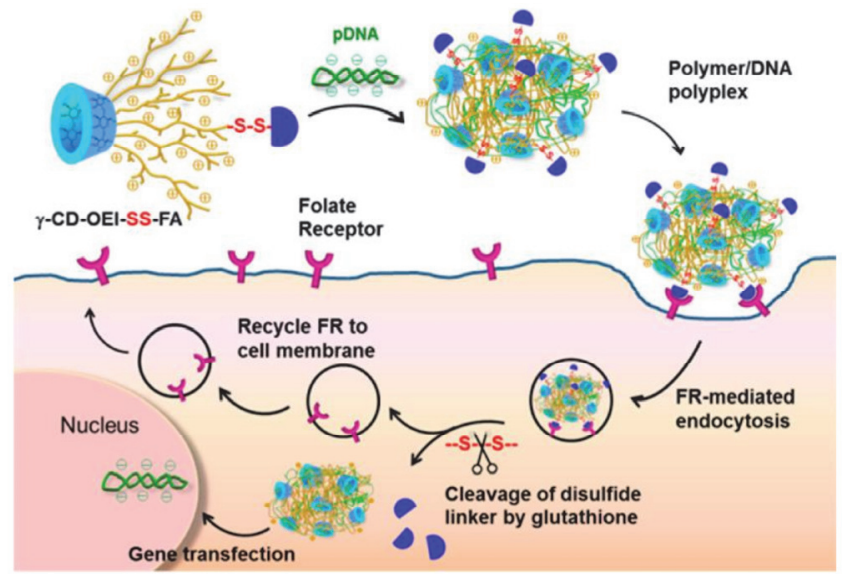

图 3 氨基环糊精构筑的基因传递体系 ${ }^{[12]}$

Figure 3 Gene delivery system by aminocyclodextrin ${ }^{[12]}$ Reproduced with permission. Copyright 2013, American Chemical Society

$\mathrm{H}_{2} \mathrm{O}_{2}$ 的存在下，该组装体可以高效光解多种有机染料 和抗生素. 作者解释在光作用下, EDA-CD/POM 促进 $\mathrm{H}_{2} \mathrm{O}_{2}$ 产生的 $\cdot \mathrm{OH}$, 而 $\cdot \mathrm{OH}$ 可以通过环糊精空腔转移到溶 液中, 在 $\cdot \mathrm{OH}$ 的作用下污染物可以降解成无害物质. 这 种超分子杂化材料可以大大增加光降解效率. 此外, 正 电荷环糊精还可以用于构筑杂化凝胶材料. 比如，胍基 $\beta$-环糊精和聚丙二醇(PPG)链通过非共价相互作用形成 超分子准聚轮烷, 将准聚轮烷与锂皇石无机黏土相互作 用就构筑了超分子水凝胶 ${ }^{[3 a]}$. 该水凝胶形成的主要驱动 力是正电荷胍基与负电荷锂皇石纳米片的静电相互作 用. 研究结果显示该水凝胶具有自愈合性能和染料负载 能力. 这种简便构筑有机-无机杂化超分子水凝胶的方 法为超分子软材料的实际应用奠定了基础.

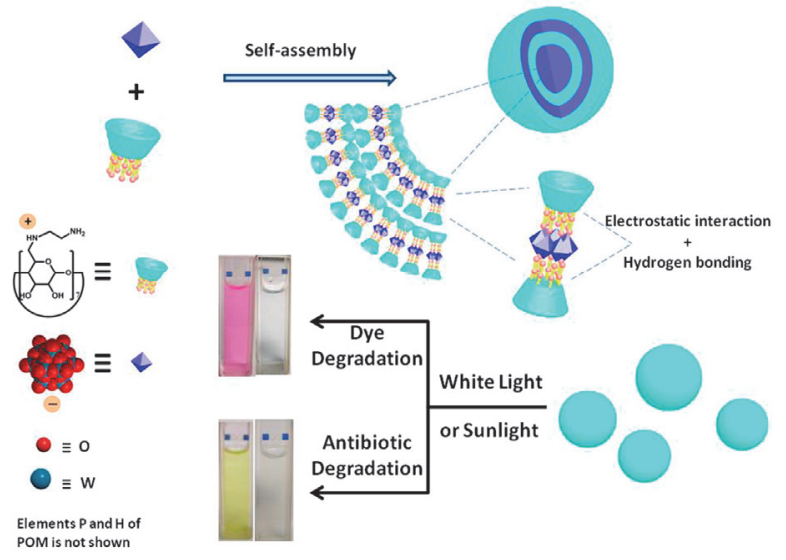

图 4 氨基环糊精构筑的光降解体系 ${ }^{[13]}$

Figure 4 Photodegradation system by aminocyclodextrin ${ }^{[13]}$ Reproduced with permission. Copyright 2019 American Chemical Society

\section{3 两亲性环糊精}

超分子囊泡一般由两亲性分子组装而成，所以是一 
种能同时负载亲水性和疏水性两种活性成分的独特体 系，这种类似于天然生物膜的性质使其被广泛用于物质 递送和生物领域 ${ }^{[14]}$. 在 2000 年, Ravoo 课题组 ${ }^{[15]}$ 报道了 首个完全由两亲环糊精组成的双层纳米囊泡体系, 该囊 泡结合了脂质体和大环主体分子的特性, 为开发先进的 主客体载体和递送系统奠定了研究基础. 在之后的研究 中发现, 两亲环糊精囊泡可以与具有多个键合位点的客 体分子进行组装. 这使得两亲环糊精构筑的软材料在纳 米功能材料领域具有举足轻重的地位 ${ }^{[16]}$. 这里我们主 要介绍两亲性多电荷环糊精.

Nishimura 等 ${ }^{[17]}$ 在 2002 年报道了硫酸盐两亲环糊精 形成的具有负电性的囊泡. 紧接着, Ravoo 课题组 ${ }^{[18]}$ 报 道了氨基两亲环糊精, 这是首次报道的在水溶液中形成 具有正电性的双层囊泡, 开启了两亲性环糊精构筑功能 超分子组装体的新篇章. 得益于囊泡体系的负载能力, 这样的组装体常作为基因传递的非病毒载体 ${ }^{[19]}$. 同时, 基于两亲性多电荷环糊精的新型功能性组装的不断发 展. 我们课题组 ${ }^{[20]}$ 合成了烷基链修饰的多咪唑正电荷 两亲性环糊精(AMCD)(图 5), 并构筑了用于抗肝素凝血 剂的新型协同治疗超分子组装体. 肝素是常用于血液透 析手术中的抗凝血剂, 使用时会引起出血并发症和功能 性维生素 $\mathrm{K}(\mathrm{VK})$ 缺乏症. 肝素的结构决定了其具有负 电荷多糖的特点, 可以与正电荷 AMCD 相互作用. 为了 测量 AMCD 和肝素未分级肝素(UFH)的键合常数, 作者 利用主客体相互作用构筑了 AMCD 和 8-差基萠-1,3,6三磺酸三钠盐(HPTS)的络合物, 通过苂光竞争滴定实 验得出键合常数为 $2.4 \times 10^{6} \mathrm{~L} \cdot \mathrm{mol}^{-1}$, 也说明该体系可 以用作 UFH 苂光传感的指示剂. 活化部分促凝血酶原

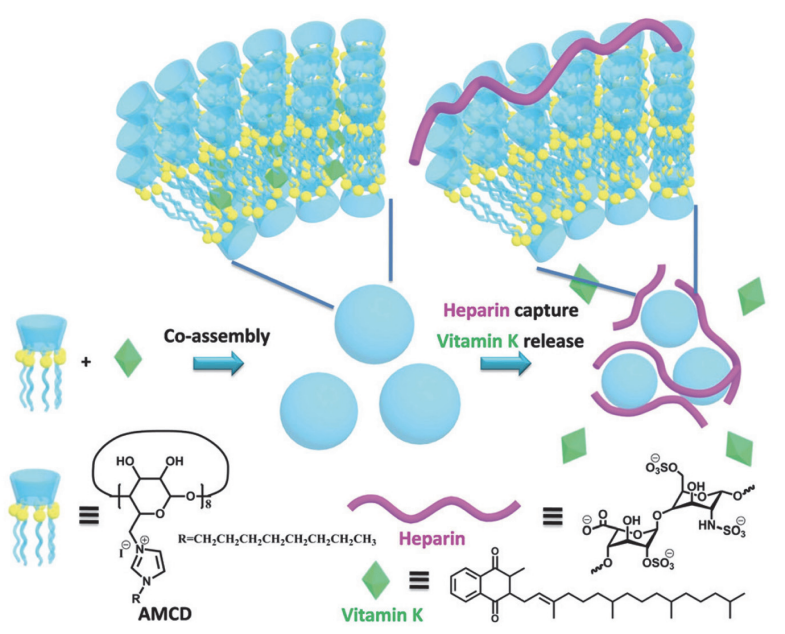

图 5 两亲环糊精构筑的协同治疗体系 ${ }^{[20]}$

Figure 5 Synergistic treatment system by amphiphilic cyclodextrin $^{[20]}$

Reproduced with permission. Copyright 2019, The Royal Society of Chemistry
激酶时间(aPTT)凝血试验证实了 AMCD 对血浆中肝素 的中和能力. 在此研究基础上, 作者构筑了 AMCD 和 $\mathrm{VK}$ 的共组装体, 该组装体可以在中和肝素过程中实现 对 $\mathrm{VK}$ 的释放. 该研究为临床手术中实现肝素中和与 VK 补充协同治疗提供了新思路.

\section{2 多电荷环糊精的智能体系}

多电荷环糊精超分子组装通过引入智能响应组分 可衍生出许多具有独特拓扑特征和特定功能的纳米刺 激响应结构, 这提供了一种有效的方式来构筑智能体 系. 超分子智能体系可以模拟多种自然和生理过程的刺 激响应结构, 适应微环境变化并且动态响应, 可应用于 各个领域. 这部分主要根据常用的刺激敏感成分来介绍 多电荷环糊精智能超分子组装体，这些成分的研究可以 了解刺激响应过程中组装体中组分的物理化学性质和 分子间聚集模式的变化, 有助于精准地设计智能组装体 结构.

\section{$2.1 \mathrm{pH}$ 响应}

生物体内 $\mathrm{pH}$ 值在不同部位会有明显的不同，例如， 正常组织 $\mathrm{pH}$ 为 7.4 , 肿瘤组织 $\mathrm{pH} \approx 6.8$, 感染和炎症部 位 $\mathrm{pH} \approx 6.5$, 而溶酶体中 $\mathrm{pH}$ 为 $4 \sim 5$. 所以 $\mathrm{pH}$ 刺激响应 水解或降解的体系对于选择性地将药物递送到器官、组 织甚至亚细胞器至关重要, 可以实现更好的疗效并避免 副作用. 一般来说, 构筑 $\mathrm{pH}$ 刺激响应的体系是将 $\mathrm{pH}$ 响 应性基团(羧基、亚胺基、硼酸基和乙酰基)或者聚合物 (聚丙烯酰胺、甲基丙烯酸二乙氨基乙酯)共组装到超分 子体系中，这些基团或聚合物在酸性条件下可被质子化 或分解, 从而导致组装体系的解离 ${ }^{[21]}$. 多电荷环糊精组 装体大多是利用静电相互作用和聚集诱导策略构筑而 成，可以方便地将上述基团或者聚合物引入到体系中从 而成功构筑智能 $\mathrm{pH}$ 响应组装体.

$\mathrm{pH}$ 变化会影响主体分子的电荷性质的变化来影响 组装体的组装模式，从而实现控制释放. 例如，本课题 组 ${ }^{[22]}$ 构筑了羧酸化环糊精/抗癌药物包合物, 研究了环 糊精主体与多种抗癌药物的键合行为. 研究结果表明在 不同 $\mathrm{pH}$ 条件下, 主体分子对抗癌药键合能力不同, 该 体系实现了对药物分子 $\mathrm{pH}$ 响应释放. 多羧酸环糊精与 抗癌药物选择性键合和控制释放行为使其有望成为抗 癌药物的新型传递体系. 另外, 羧酸环糊精和季铵盐二 茂铁衍生物构筑的 $\mathrm{pH}$ 响应超分子组装体 ${ }^{[23]}$, 也是由于 主体在酸性条件下会质子化失去电性导致体系解组装 从而实现抗癌药盐酸阿霉素(DOX)的有效释放. 除此之 外, 聚轮烷(PR)这种超分子结构也常用于构筑智能纳米 组装体. Caruso 等 ${ }^{[24]}$ 报道了一种 $\mathrm{pH}$ 响应性的负电荷聚 轮烷纳米粒子. 如图 6 , 选择一种由 $\alpha-\mathrm{CD}$ 和聚乙二醇 
(PEG)构筑的低覆盖度聚轮烷(PRX 5)来确保 CD 在高分 子链上高迁移率, 其中 $\mathrm{CD}$ 被响应性羧酸基团修饰后, 在酸性的条件下 PRX5 自组装成稳定的纳米粒子, 作者 认为纳米颗粒的形成可能来源于 $\mathrm{CD}$ 的羧酸部分和羟基 基团之间的分子内和分子间氢键. 当 $\mathrm{pH}$ 上升时, 由于 各个 PRX 上带负电荷的羧酸根之间的排斥导致纳米粒 子发生解离, 且该响应过程具有一定的重复性. 研究还 证明了 $\mathrm{CD}$ 的迁移率为关键因素, 因为具有较高覆盖率 PRX 的酸化不会形成组装体.

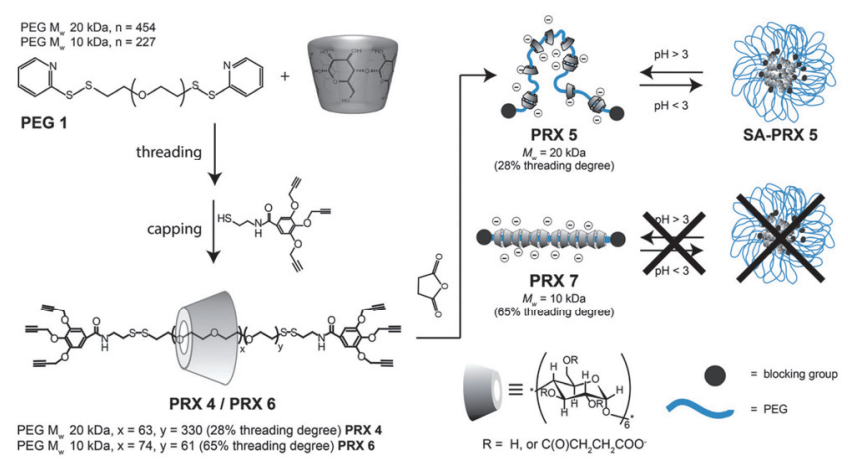

图 6 多电荷环糊精构筑的 $\mathrm{pH}$ 响应聚轮烷体系 ${ }^{[24]}$

Figure 6 pH-responsive polyrotaxane system by multicharged $^{[24]}$

Reproduced with permission. Copyright 2016, The Royal Society of Chemistry

$\mathrm{pH}$ 变化也会通过影响客体分子的电荷性质使组装 体的组装模式发生改变. 比如, 磺化环糊精和聚丙烯酰 胺(PEI)构筑的 $\mathrm{pH}$ 响应 ATP 超分子智能载体 ${ }^{[25]}$, 由于 $\mathrm{pH}$ 影响 PEI 的质子化程度, 因此组装体表现出良好的 $\mathrm{pH}$ 响应组装/解组装行为以及对 ATP 的负载/持续释放 能力. 对于口服药物一般是希望在胃酸性环境中保持稳 定而在肠碱性中释放. 这就要求药物载体在不同 $\mathrm{pH}$ 下 实现药物的可控释放, 而新型智能纳米粒子是解决这个 问题的突破口. 基于此, 本课题组 ${ }^{[26]}$ 报道了磺化环糊精 和壳聚糖(CS)构筑的 $\mathrm{pH}$ 响应智能纳米粒子(图 7). 研究 结果表明球形纳米粒子在室温下稳定存在不会发生解 聚. 由于构筑模块具有离子结构, $\mathrm{pH}$ 的变化可以控制超 分子组装体的组装与解组装, 当 $\mathrm{pH}$ 从 5.3 到 10.4 可以 实现纳米粒子的解组装, 当 $\mathrm{pH}$ 调回到 5.3 时纳米粒子重 新组装. 在这个研究基础上, 作者利用 $\mathrm{SCD} / \mathrm{CS}$ 体系负 载了消化道的抑菌药物黄连素(BE), 研究表明当 $\mathrm{pH}$ 值 从 2(即胃的环境 $\mathrm{pH}$ )增加到 8 (即肠的环境 $\mathrm{pH}$ )时, 负载 $\mathrm{BE}$ 的释放效率明显增加. 动物实验进一步证实了该纳 米粒子负载药物在经过胃时保持稳定而在肠道中进行 释放. 该体系有望用于药物分子的精准释放.

\section{2 酶响应}

相比于其他刺激响应的智能体系, 酶响应体系具有

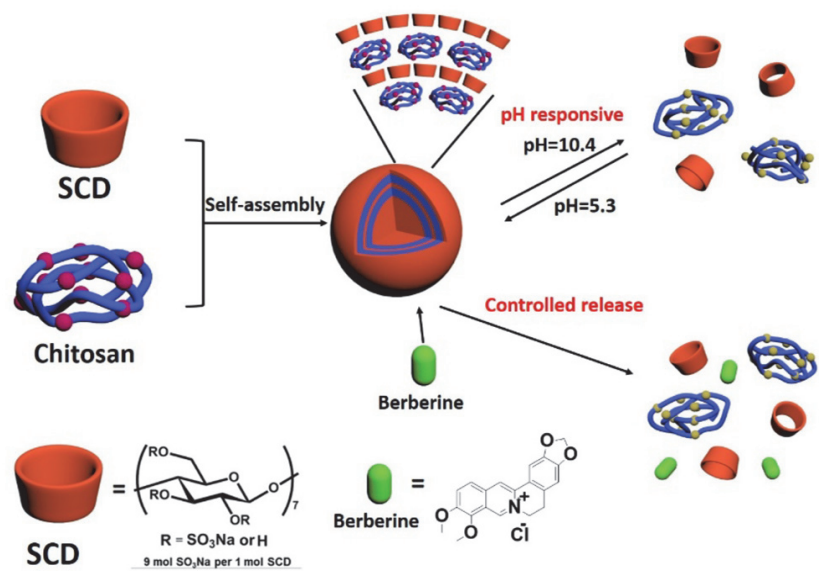

图 7 磺化环糊精/壳聚糖构筑的 $\mathrm{pH}$ 响应体系 ${ }^{[26]}$

Figure 7 pH-responsive system by sulfonated cyclodextrin/ chitosan $^{[26]}$

Reproduced with permission. Copyright 2018, American Chemical Society

良好的生物相容性、高度选择性和可控降解的优势，一 直是智能响应体系研究的热点. 同时生物体内所有生命 活动最终受酶的控制，也包括特定的疾病. 多电荷环糊 精因其优异的生物相容性以及空腔特异性结合底物的 性质，常作为构筑酶响应体系的理想主体. 在环糊精体 系中引入酶响应位点或者引入特定疾病相关酶用于靶 向治疗是构建酶响应超分子纳米组装体的主要思路 ${ }^{[27]}$. 常见酶响应体系的构筑主要利用鱼精蛋白对胰蛋白酶、 肉豆冦酰胆碱和酯键对丁酰胆碱酯酶、透明质酸对透明 质酸酶的特异性响应.

将酶响应酯键引入多电荷环糊精主体分子中是酶 响应智能体系的一种构筑方法. 如图 8 所示，以具有七 个可裂解酯键的咪唑鎓 $\beta-\mathrm{CD}$ 衍生物(EICD)为主体分子,

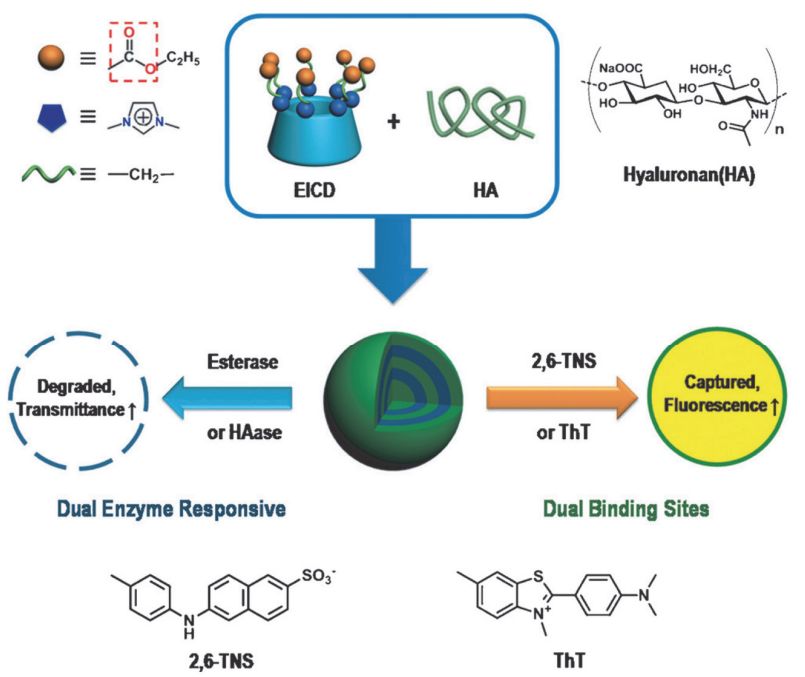

图 $8 \mathrm{EICD} / \mathrm{HA}$ 构筑的酶响应体系 ${ }^{[28]}$

Figure 8 Enzyme-responsive system by EICD/HA ${ }^{[28]}$ Reproduced with permission. Copyright 2016, Wiley-VCH 
通过 EICD 和透明质酸(HA)静电相互作用构筑了超分子 组装体 ${ }^{[28]}$. 该超分子组装体具有双重酶响应性, 人工羧 酸酯对于乙酰胆碱酯酶的响应和透明质酸对透明质酸 酶的响应, 并且透明质酸可以定向靶向癌细胞. 同时由 于 EICD 空腔可以结合负电荷底物, 而 EICD/HA 体系可 以负载正电荷底物, 该体系具有正电性和负电性底物负 载能力. 双酶响应和双负载能力的结合使该体系具有靶 向癌细胞和高效释放多种药物的能力.

基于客体分子酶刺激响应的超分子组装体常被用 于控制释放. 我们课题组 ${ }^{[29]}$ 利用带负电的磺化环糊精 (SCD) 与鱼精蛋白构筑了生物相容性优异的新型胰蛋白 酶响应性的超分子组装体. 由于鱼精蛋白可以特异性响 应肤蛋白酶, 当向组装体溶液中加入肤蛋白酶可以破坏 $\mathrm{SCD}$ /鱼精蛋白纳米粒子从而促进负载药物模型分子的 释放. 该超分子组装体提供了一种酶响应型药物控制释 放的智能模型. 此外, 羧酸 $\beta$-CD 诱导氯化肉豆冦酰胆 碱聚集形成的组装体 ${ }^{[30]}$. 主客体通过静电相互作用形 成球形纳米粒子, 并且在丁酰胆碱酷酶的作用下纳米粒 子能够实现对药物模型分子的控制释放. 另一个典型例 子是磺化环糊精诱导聚集前药分子胆碱修饰的苯丁酸 氮芥 (QA-Cbl)形成的组装体 ${ }^{[31]}$ (如图 9). 客体分子可以 被丁酰胆碱酯酶特异性切断并释放抗癌药物苯丁酸氮 芥 $(\mathrm{Cbl})$. 这种利用静电相互作用构筑的纳米粒子安全 无毒, 而且前药直接作为客体分子大大提高了药物的负 载率. 在丁酰胆碱酯酶作用下组装体解组装并且释放活 性抗癌药物. 该高效酶响应的智能超分子组装体利用多 电荷大环/前药的组装模式, 为解决癌症治疗中多药耐 药性的问题提出新方法.

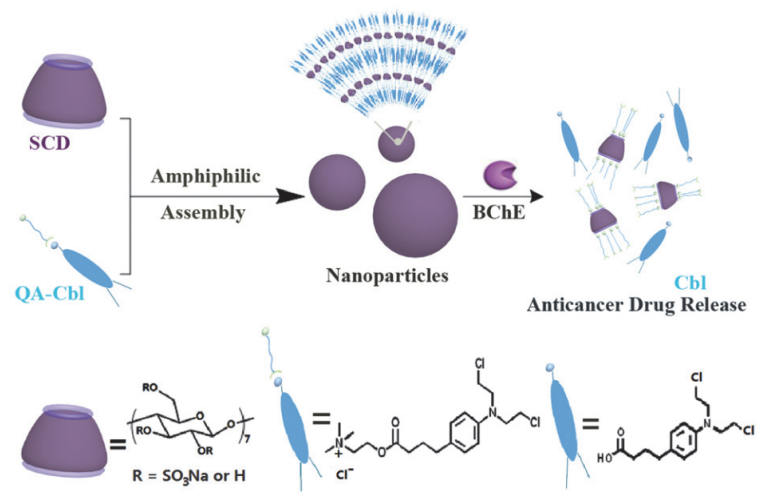

图 9 磺化环糊精/前药构筑的酶响应体系 ${ }^{[31]}$

Figure 9 Enzyme-responsive system by sulfatocyclodextrin/prodrug ${ }^{[31]}$

Reproduced with permission. Copyright 2019. The Royal Society of Chemistry

\section{3 光响应}

在多种类型的刺激响应中, 光因其远程可控、非侵
入性、环保性和瞬时性等优势赋予光刺激响应体系巨大 的应用价值. 光响应体系的构筑离不开光敏基团，如偶 氮苯类、香豆素类和葱等.一直以来，基于特定光敏基 团的光诱导异构化、降解和聚合等性质构筑了各种各样 的光响应智能体系 ${ }^{[16 e, 32]}$. 因此, 将光敏基团引入到超分 子纳米载体中就可以利用超分子策略实现精确地组装 以及特定时间和特定空间的光刺激智能响应.

例如，利用磺化环糊精和两亲性葱衍生物通过静电 作用构筑光响应降解的超分子组装体 ${ }^{[33]}$, 该组装体提 供的疏水环境有利于葱环的进一步堆积, 从而在光照条 件下产生单线态氧, 加速葱衍生物的光降解速率. 本工 作为利用超分子策略构筑多环芳烃的光解体系以及光 敏材料体系提供了思路. 我们课题组 ${ }^{[34]}$ 报道了咪唑环 糊精(ICD)与磺化偶氮苯衍生物 (G)构筑的光响应超分子 组装体(图 10). 单独客体分子具有两亲性可以自组装成 小的纳米粒子, 加入咪唑 $\beta$ - $\mathrm{CD}$ 后被诱导聚集成较大的 纳米粒子. 而且, 该超分子组装体可以通过静电相互作 用和 $\pi-\pi$ 相互作用稳定地负载染料和药物. 更重要的是, 在紫外光照射下, 偶氮苯分子由 trans $-\mathrm{G}$ 转变为 $c i s-\mathrm{G}$, 导致组装体解体重组, 使纳米粒子体积变小. 在可见光 照射后，又可以可逆地恢复到之前的组装模式. 同时实 验结果表明此纳米粒子光响应可逆变化具有良好的往 复性. 这种光控组装与解组装可以有效实现组装体中负 载物阿霉素的可控释放. 所以, 这种光响应的智能超分 子组装体有望作为纳米载体应用于药物传递和可控释 放, 减少抗癌药物的副作用并提高其生物利用度.

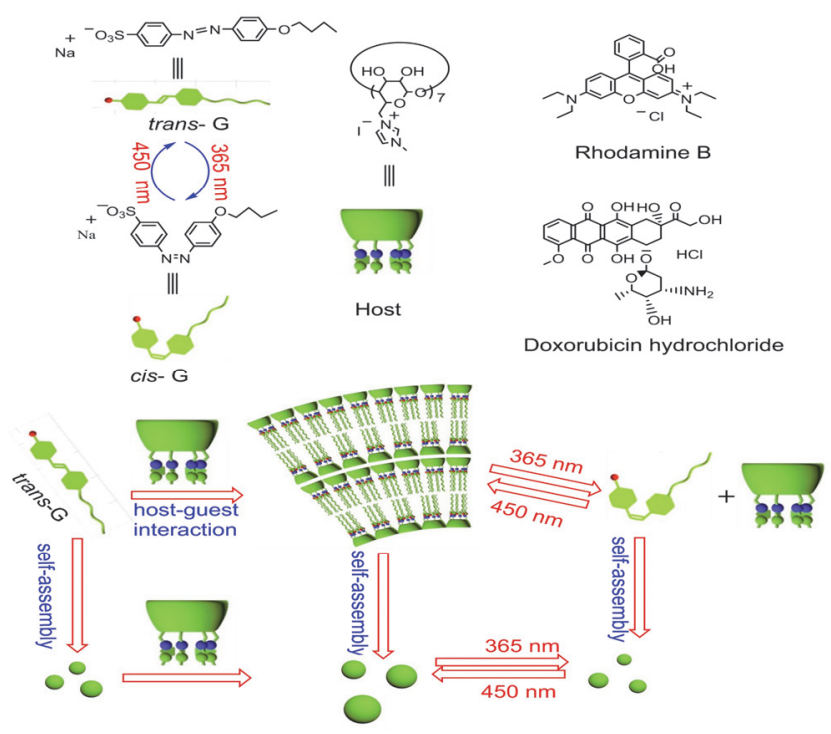

图 10 咪唑环糊精/偶氮苯构筑的光响应体系 ${ }^{[34]}$

Figure 10 Photo-response system by imidazole cyclodextrin/ azobenzene ${ }^{[34]}$

Reproduced with permission. Reproduced with permission. Copyright 2018, Wiley-VCH 
牛忠伟等 ${ }^{[35]}$ 通过主体分子环糊精上氨基 $\left(\mathrm{NH}_{2}\right)$ 和疏 水性聚乳酸(PLA)末端羧基(COOH)之间的静电相互作 用构筑了一种两亲超分子组装体. 组装体在水中呈现的 是以环糊精为壳的核壳结构. 所以作者利用 $\mathrm{CD}$ 空腔包 结光响应型分子偶氮苯衍生物, 赋予了组装体智能光刺 激响应性质. 实验结果证实了在紫外光和可见光的交替 照射下, 组装体纳米结构的大小可以往复性改变. 此外 还研究了 $\mathrm{CD}$ 空腔对探针分子 8-苯胺-1-萗磺酸盐(ANS) 的包结以及组装体对阿霉素的负载和 $\mathrm{pH}$ 响应释放行为.

\section{4 氧化还原响应}

同时, 也有许多研究致力于探索具有氧化还原敏感 性基团的纳米体系. 最常用的方法是将还原敏感的二硫 键或氧化一还原响应二茂铁基团引入到环糊精体系中, 为开发氧化还原响应纳米体系并应用于基因治疗提供 了条件 ${ }^{[36]}$.

利用二硫键将靶向剂等功能基团连接到核心 $\mathrm{CD}$ 上 形成的主体分子可以用于构筑具有氧化还原响应性的 超分子基因载体. 比如, 将靶向基团叶酸(FA)通过二硫 键连接到多氨基取代的 $\beta$ - $\mathrm{CD}$ 上作为主体化合物 ${ }^{[37]}$. 如 图 11 所示, 通过主客体作用将抗癌药紫杉醇包封入 $\mathrm{CD}$ 腔中, 并与质粒 DNA 静电相互作用构筑了纳米粒子. FA 诱导纳米粒子靶向肿瘤, 二硫键响应细胞内谷胱甘 肽, 释放 FA 分子, 促进叶酸受体的循环, 进一步提高转 染效率. 结果表明, 该纳米超分子体系对肿瘤生长有明 显的抑制作用. 此外, 还有一种以 $\mathrm{CD}$ 为核心的星形阳 离子聚合物与金刚烷改性离子聚合物通过主客体相互 作用形成的多功能基因载体体系 ${ }^{[38]}$. 体系中, 主体聚合 物 $(\beta$-CD-SS-P) 是通过二硫键连接的聚(甲基丙烯酸 2-二 甲基氨基乙酯)(pDMAEMA) 环糊精, 客体聚合物 (Ad-pMPC) 是用一个金刚烷封端的聚(2-甲基丙烯酰氧 基乙基磷酸胆碱)(pMPC). Ad-pMPC 赋予体系胞外稳定 性和血清耐受性并且可以提高细胞摄取能力. 同时, 在 GSH 存在的情况下, 环糊精上二硫键的还原响应使体 系将逐渐解组装, 从而触发负载 DNA 的释放. 该体系 可以显著增强基因转染效率. 作者认为该组装体优异的 基因传递能力可用于癌症的治疗.

除此之外，聚轮烷(PR)这种超分子结构也常用于构 筑还原响应智能纳米组装体. 在之前的报道中就曾研究 过由正电荷环糊精构成的聚轮烷, 并评估了其与 DNA 络合以及体外转染能力 ${ }^{[39]}$. 比较有代表性的是由含多 个正电荷的 $\alpha$-环糊精和含二硫键的聚乙二醇(PEG)组成 的可生物裂解的聚轮烷 ${ }^{[0]}$. 该超分子组装体具有稳定 的正电荷, 可作为非病毒基因载体, 而二硫键的引入为 其提供了在细胞还原环境中的可降解性. 作者研究发现 聚轮烷的这种结构有助于增强基因传递效率. 一个典型

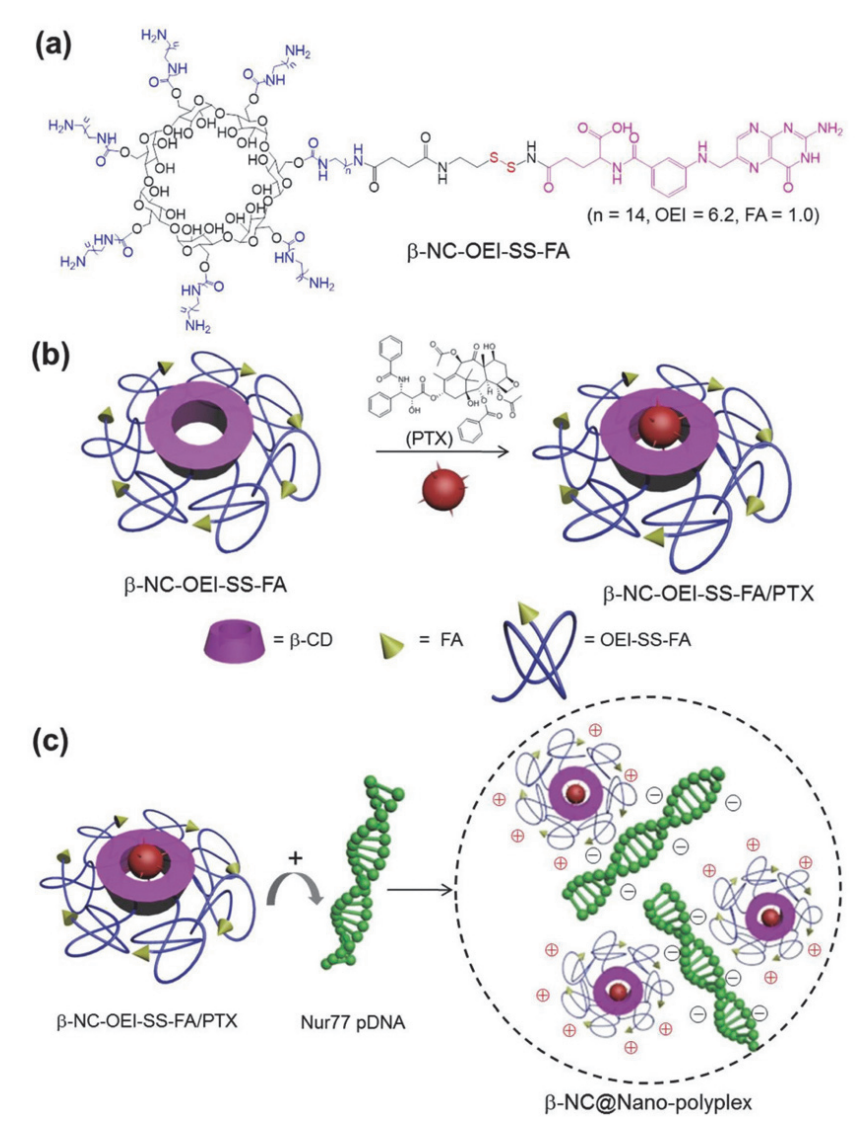

图 11 氨基环糊精构筑的氧化还原响应体系 ${ }^{[37]}$

Figure 11 Redox-response system by aminocyclodextrin ${ }^{[37]}$ Reproduced with permission. Copyright 2016, The Royal Society of Chemistry

的例子是将由 $\mathrm{N}, \mathrm{N}$-二甲基氨基乙基(DMAE)基团修饰的 $\alpha-\mathrm{CD}$ 穿入 $\mathrm{PEG}$ 轴上并用二硫键引入封端基团构成正电 荷聚轮烷 ${ }^{[41]}$, 该聚轮烷具有 GSH 还原响应性, 可用于 siRNA 的递送. 研究发现聚轮烷上 $\alpha$-CD 数量的不同会 影响 PR/siRNA 复合物对聚阴离子竞争的抵抗力. 结果 表明, 具有 52 个 $\alpha$-CD 的 PR 可以高度稳定地凝聚 siRNA, 并在细胞内 GSH 的作用下将其释放. 因此作者 认为该超分子智能组装体可用于 siRNA 传递与治疗.

\section{5 磁响应}

除了上面提到几种比较典型的刺激响应类型, 磁响 应材料也因其在自然现象和生理活动中的重要作用而 受到研究者的广泛关注 ${ }^{[42]}$. 将多电荷环糊精引入到以 磁纳米粒子为核心的磁性材料中, 再利用非共价相互作 用赋予磁纳米体系相应的功能, 就可以构筑具有特殊功 能并可以高度靶向外部磁场部位的磁响应超分子组装 体. 磁性氧化铁纳米粒子作为磁性材料最常用的构筑原 料有望应用于物质分离和生物治疗领域 ${ }^{[3]}$.

Ravoo 课题组 ${ }^{[44]}$ 报道了一个简单的动态超分子系 统, 可以在磁场中选择性地从蛋白质混合物中捕获和分 离所需的蛋白质. 如图 12, 作者将羧酸化环糊精修饰到 
磁纳米粒子表面，利用主客体相互作用将金刚烷糖类衍 生物负载到超分子纳米粒子上, 从而功能化磁纳米粒 子, 客体分子上的糖类可以特异性地与蛋白质结合. 该 磁响应纳米粒子为蛋白质的选择性吸附提供了非常大 的有效表面积, 并且通过施加外部磁场使纳米粒子与溶 液分离而且不会造成蛋白质变性, 实现了对混合蛋白质 的高效分离. 该研究促进了超分子纳米体系对特定蛋白 质的捕获和分离.
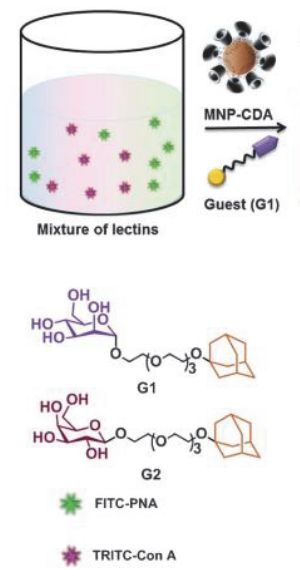

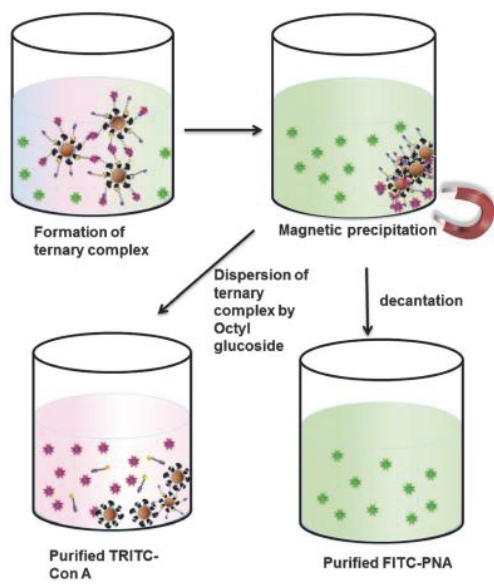

图 12 羧酸环糊精构筑的磁响应体系 ${ }^{[44]}$

Figure 12 Magnetic-response system by carboxycyclodex$\operatorname{trin}^{[44]}$

Reproduced with permission. Copyright 2014, Wiley-VCH

全氟化合物(PFCs)和重金属是新兴污染物, 这些有 害物质的清除也受到了极大关注. 比如, Suri 课题组 ${ }^{[43 a]}$ 报道了一种基于环糊精分子的多功能磁性吸附剂, 用于 去除水溶液中的全氟辛烷磺酸( $\mathrm{PFOS}$ )、全氟辛酸(PFOA) 和 $\mathrm{Cr}(\mathrm{VI})$. 环糊精的引入使纳米粒子可以利用疏水相互 作用和静电相互作用吸附 PFC 和 $\mathrm{Cr}(\mathrm{VI})$. 此外, 该纳米 粒子的回收和重复使用可以通过外加磁场进行控制, 重 复使用次数高达 10 次且无任何明显的效率损失.

\section{6 多重刺激响应}

为了最大限度提高多电荷环糊精智能组装体的功 能性和利用率, 研究者考虑将多种刺激集成到环糊精纳 米平台体系中, 这样的体系更方便灵活和智能, 应用领 域更加广泛，可表现出协同效应和协同增强作用.

Singh 等 ${ }^{[45]}$ 研究了磺化 $\beta$-环糊精诱导聚集染料金胺 $\mathrm{O}(\mathrm{AuO})$ 客体分子形成的聚集体. 仔细研究了该聚集体 的光物理行为, 发现在 SCD 的高电荷环境下, $\mathrm{AuO}$ 显示 强烈荧光发射和更长的荧光寿命, 并且最大发射红移至 $560 \mathrm{~nm}$. 该智能超分子聚集体对介质的温度和离子强度 等外界刺激具有灵敏的响应性. 最近他们 ${ }^{[46]}$ 还报道了 硫酸化的 $\beta$-CD 诱导有机荧光探针芘的氨基衍生物 (PMA)自聚集的超分子体系. 带负电荷的硫酸盐基团对
带正电荷探针的电荷中和以及环糊精边缘的紧密接近, 诱导了 PMA 分子的基态缔合. 并且这种单体一二聚体平 衡体系对外部化学刺激(例如温度、 $\mathrm{pH}$ 、介质的离子强 度和有机溶剂)具有极强的响应能力. 研究表明该超分 子组装体可检测精氨酸, 有望用于传感检测.

还有一个典型的例子是基于正电荷咪唑 $\beta-\mathrm{CD}$ 的超 分子体系具有可控 DNA 凝聚能力 ${ }^{[47]}$. 如图 13 所示, 合 成了由不同长度疏水链连接的咪唑鎓与羧酸酯作为修 饰基团的全取代 $\beta$ - $\mathrm{CD}$, 主体分子的正电性决定了其良 好的 DNA 凝聚能力. 研究表明在碱或酶的存在下, 由 主体分子的酯水解产生了羧酸根, 此时电性变化导致 DNA 凝聚能力变弱. 而且发现疏水链的长度影响 DNA 凝聚能力及环糊精/DNA 体系的稳定性, 疏水链越长, CD 对 DNA 凝聚能力越弱. 随后研究了主体分子的细胞 毒性，结果证明该基因载体具有良好的生物相容性. 该 体系通过调节基因载体的电荷类型和分布来实现酶和 $\mathrm{pH}$ 双调控 DNA 的凝聚和释放, 有望作为高效的基因传 递载体.

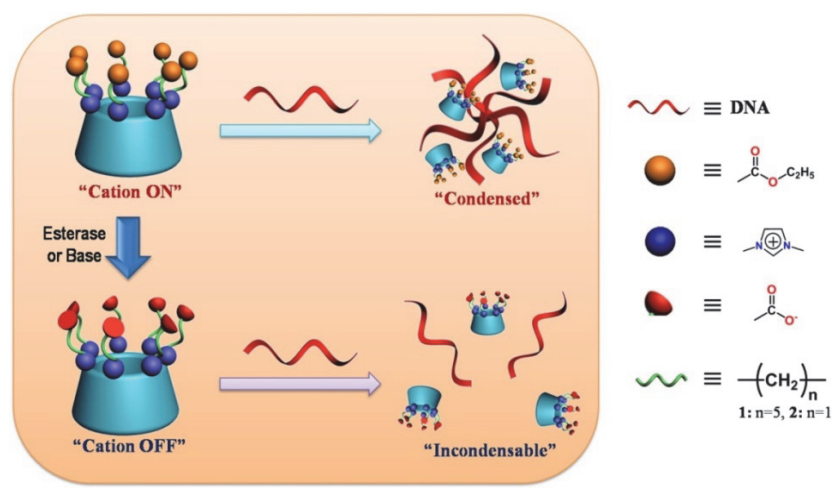

图 13 多电荷环糊精构筑的酶和 $\mathrm{pH}$ 双响应体系 ${ }^{[47]}$

Figure 13 Enzyme and $\mathrm{pH}$ response system by multi-charge cyclodextrin $^{[47]}$

Reproduced with permission. Copyright 2015, The Royal Society of Chemistry

\section{3 总结与展望}

综上所述，通过一些典型的例子总结了多电荷环糊 精智能组装体最新研究进展. 从主体类型和智能组装体 多种刺激响应模式的方面来进行介绍，阐述了多电荷环 糊精通过分子诱导聚集构筑超分子纳米结构带来的智 能响应性的机理，尤其是引入多种刺激响应基团之后体 现了智能组装体优异的生物相容性、可控的生物降解 性、高效的负载能力和精准的底物调节释放功能, 表明 了该智能组装体可以应用于药物和基因传递、控制释 放、癌症治疗和传感检测等领域. 基于 $\mathrm{CD}$ 的智能体系 已被经常用于多类型物质传递的纳米平台, 在精准递送 领域存在巨大潜力. 提高多电荷环糊精智能组装体环境 的实时响应性和靶向的精准递送效率, 构建高效的纳米 
递送平台，对于更好地拓展其智能应用的领域，仍然充 满了机遇和挑战.

目前主体分子修饰方法单一, 所以多电荷环糊精的 类型也比较固定, 导致主体分子的识别能力比较有限, 超分子体系的应用相对局限. 下一步应该致力于开发新 的修饰方法, 对 CD 骨架进行区域选择性修饰, 通过修 饰基的功能和环糊精的不同空腔尺寸来提高主体对客 体分子的选择性键合, 开拓多电荷环糊精在化学、生物 以及材料领域的应用.

环糊精中多电荷的引入可以通过静电相互作用极 大促进智能纳米超分子体系的构筑. 因此, 利用主体与 多种客体共组装超分子体系可以增加多种非共价键作 用力的协同效应, 提高多电荷环糊精超分子组装体的综 合性能, 可为构筑多重刺激响应的超分子组装体提供新 思路.

尽管天然环糊精和多电荷环糊精本身是无毒或低 毒的, 然而其与客体分子共组装形成的环糊精智能体系 在药物和基因传递以及疾病治疗方面仍存在安全性问 题. 因此, 低毒高效的纳米智能体系是未来发展的重要 方向.

\section{References}

[1] (a) Crini, G. Chem. Rev. 2014, 114, 10940.

(b) Chen, Y.; Zhang, Y. M.; Liu, Y. Chem. Commun. 2010, 46, 5622. (c) Lai, W.-F.; Rogach, A. L.; Wong, W.-T. Chem. Soc. Rev. 2017, 46, 6379 .

(d) Prochowicz, D.; Kornowicz, A.; Lewiński, J. Chem. Rev. 2017, 117, 13461.

(e) Chen, G.; Jiang, M. Chem. Soc. Rev. 2011, 40, 2254.

(f) Chen, H.; Liu, X.; Dou, Y.; He, B.; Liu, L.; Wei, Z.; Li, J.; Wang, C.; Mao, C.; Zhang, J.; Wang, G. Biomaterials 2013, 34, 4159.

(g) Schmidt, B.; Barner-Kowollik, C. Angew. Chem., Int. Ed. 2017, 56,8350 .

(h) Kolesnichenko, I. V.; Anslyn, E. V. Chem. Soc. Rev. 2017, 46, 2385

(i) Chen, Y.; Liu, Y. Chem. Soc. Rev. 2010, 39, 495

(j) Zhang, Y.; Liang, L.; Chen, Y.; Chen, X. M.; Liu, Y. Soft Matter 2018, 15,73 .

(k) Yu, Q.; Zhang, Y.-M.; Liu, Y.-H.; Xu, X.; Liu, Y. Sci. Adv. 2018, 4, eaat2297.

(1) Pinalli, R.; Pedrini, A.; Dalcanale, E. Chem. Soc. Rev. 2018, 47, 7006.

(m) Zhang, W.; Deng, W. Chin. J. Org. Chem. 2018, 38, 3002 (in Chinese).

(张薇, 邓维, 有机化学, 2018, 38, 3002.)

(n) Chen, Y. Q.; Gui, X.; Duan, Z. B.; Zhu, L. J.; Xiang, Y. Z.; Xia, D. H. Chin. J. Org. Chem. 2019, 39, 1284 (in Chinese).

(陈雅琪，桂金金，段尊斌，朱丽君，项玉芝，夏道宏，有机化学， 2019, 39, 1284.)

(o) Qie, S. Y.; Hao, Y.; Liu, Z. J.; Wang, J.; Xi, J. N. Acta Chim. Sinica 2020, 78, 232 (in Chinese).

(郄淑燕, 郝芗, 刘宗建, 王锦, 席家宁, 化学学报, 2020, 78, 232.)

[2] Eliseev, A. V.; Schneider, H.-J. J. Am. Chem. Soc. 1994, 116, 6081.

[3] (a) Li, Z.; Zhang, Y.-M.; Wang, H.-Y.; Li, H.; Liu, Y. Macromolecules 2017, 50, 1141.

(b) Zhang, Y.; Chen, Y.; Li, J.; Liang, L.; Liu, Y. Acta Chim. Sinica
2018, 76, 622 (in Chinese)

(张依, 陈湧, 李晶晶, 梁璐, 刘育, 化学学报, 2018, 76, 622.)

(c) Niu, J.; Chen, Y.; Liu, Y. Chin. J. Org. Chem. 2019, 39, 151 (in Chinese).

(牛杰, 陈湧, 刘育, 有机化学, 2019, 39, 151.)

[4] (a) Eliseev, A. V.; Schneider, H.-J. Angew. Chem., Int. Ed. 1993, 32, 1331.

(b) Steffen, A.; Thiele, C.; Tietze, S.; Strassnig, C.; Kämper, A.; Lengauer, T.; Wenz, G.; Apostolakis, J. Chem.-Eur. J. 2007, 13, 6801.

[5] (a) Zhang, Y. M.; Liu, Y. H.; Liu, Y. Adv. Mater. 2020, 32, e1806158.

(b) Liao, R.; Lv, P.; Wang, Q.; Zheng, J.; Feng, B.; Yang, B. Biomater. Sci. 2017, 5, 1736.

(c) Yao, X.; Mu, J.; Zeng, L.; Lin, J.; Nie, Z.; Jiang, X.; Huang, P. Mater. Horiz. 2019, 6, 846 .

[6] (a) Bom, A.; Bradley, M.; Cameron, K.; Clark, J. K.; Van Egmond, J.; Feilden, H.; Maclean, E. J.; Muir, A. W.; Palin, R.; Rees, D. C.; Zhang, M.-Q. Angew. Chem., Int. Ed. 2002, 41, 265.

(b) Adam, J. M.; Bennett, D. J.; Bom, A.; Clark, J. K.; Feilden, H.; Hutchinson, E. J.; Palin, R.; Prosser, A.; Rees, D. C.; Rosair, G. M.; Stevenson, D.; Tarver, G. J.; Zhang, M.-Q. J. Med. Chem. 2002, 45, 1806.

[7] Jones, S. T.; Cagno, V.; Janeček, M.; Ortiz, D.; Gasilova, N.; Piret, J.; Gasbarri, M.; Constant, D. A.; Han, Y.; Vuković, L.; Král, P.; Kaiser, L.; Huang, S.; Constant, S.; Kirkegaard, K.; Boivin, G.; Stellacci, F.; Tapparel, C. Sci. Adv. 2020, 6, eaax9318

[8] Sun, H.-L.; Zhang, Y.-M.; Chen, Y.; Liu, Y. Sci. Rep. 2016, 6, 27.

[9] Li, J. J.; Chen, Y.; Yu, J.; Cheng, N.; Liu, Y. Adv. Mater. 2017, 29, 1701905.

[10] (a) Shi, R.-J.; Chen, Y.; Hou, X.-F.; Liu, Y. RSC Adv. 2016, 6, 15175

(b) Zhao, D.; Chen, Y.; Liu, Y. Chin. Chem. Lett. 2015, 26, 829

[11] Díaz-Moscoso, A.; Balbuena, P.; Gómez-García, M.; Ortiz Mellet, C.; Benito, J. M.; Le Gourriérec, L.; Di Giorgio, C.; Vierling, P.; Mazzaglia, A.; Micali, N.; Defaye, J.; García Fernández, J. M. Chem. Commun. 2008, 2001.

[12] Zhao, F.; Yin, H.; Zhang, Z.; Li, J. Biomacromolecules 2013, 14 476.

[13] Wang, J.; Chen, Y.; Cheng, N.; Feng, L.; Gu, B.-H.; Liu, Y. ACS Appl. Bio Mater. 2019, 2, 5898.

[14] (a) Lu, Y.; De Vries, W. C.; Overeem, N. J.; Duan, X.; Zhang, H.; Zhang, H.; Pang, W.; Ravoo, B. J.; Huskens, J. Angew. Chem., Int. Ed. 2019, 58, 159.

(b) Schibilla, F.; Holthenrich, A.; Song, B.; Linard Matos, A. L.; Grill, D.; Rota Martir, D.; Gerke, V.; Zysman-Colman, E.; Ravoo, B. J. Chem. Sci. 2018, 9, 7822.

(c) Himmelein, S.; Lewe, V.; Stuart, M. C. A.; Ravoo, B. J. Chem. Sci. 2014, 5, 1054.

[15] Ravoo, B. J.; Darcy, R. Angew. Chem., Int. Ed. 2000, 39, 4324.

[16] (a) Mohan Nalluri, S. K.; Bultema, J. B.; Boekema, E. J.; Ravoo, B. J. Chem. Sci. 2011, 2, 2383.

(b) Samanta, A.; Ravoo, B. J. Chem.-Eur. J. 2014, 20, 4966.

(c) Samanta, A.; Stuart, M. C. A.; Ravoo, B. J. J. Am. Chem. Soc. 2012, 134, 19909.

(d) Nalluri, S. K. M.; Bultema, J. B.; Boekema, E. J.; Ravoo, B. J. Chem.-Eur. J. 2011, 17, 10297.

(e) Moratz, J.; Stricker, L.; Engel, S.; Ravoo, B. J. Macromol. Rapid Commun. 2018, 39, 1700256

[17] Sukegawa, T.; Furuike, T.; Niikura, K.; Yamagishi, A.; Monde, K.; Nishimura, S.-I. Chem. Commun. 2002, 430.

[18] Donohue, R.; Mazzaglia, A.; Ravoo, B. J.; Darcy, R. Chem. Commun. 2002, 2864.

[19] (a) O'mahony, A. M.; Doyle, D.; Darcy, R.; Cryan, J. F.; O'driscoll, C. M. Eur. J. Pharm. Sci. 2012, 47, 896.

(b) Méndez-Ardoy, A.; Guilloteau, N.; Di Giorgio, C.; Vierling, P.; Santoyo-González, F.; Ortiz Mellet, C.; García Fernández, J. M. J. Org. Chem. 2011, 76, 5882. 
[20] Li, P. Y.; Chen, Y.; Chen, C. H.; Liu, Y. Chem. Commun. 2019, 55, 11790.

[21] (a) Zan, M.; Li, J.; Luo, S.; Ge, Z. Chem. Commun. 2014, 50, 7824. (b) Zhao, D.; Yi, X.; Xu, J.; Yuan, G.; Zhuo, R.; Li, F. J. Mater. Chem. B 2017, 5, 2823.

(c) Durmaz, Y. Y.; Lin, Y.-L.; Elsayed, M. E. H. Adv. Funct. Mater. 2013, 23, 3885 .

[22] Cheng, J. G.; Yu, H. J.; Chen, Y.; Liu, Y. Bioorg. Med. Chem. 2018, 26, 2287.

[23] Cheng, J.-G.; Zhang, Y.-M.; Liu, Y. ChemNanoMat 2018, 4, 758.

[24] Tardy, B. L.; Tan, S.; Dam, H. H.; Ejima, H.; Blencowe, A.; Qiao, G. G.; Caruso, F. Nanoscale 2016, $8,15589$.

[25] Liang, L.; Chen, Y.; Chen, X.-M.; Zhang, Y.; Liu, Y. Chin. Chem. Lett. 2018, 29, 989.

[26] Chen, X. M.; Chen, Y.; Hou, X. F.; Wu, X.; Gu, B. H.; Liu, Y. ACS Appl. Mater. Interfaces 2018, 10, 24987.

[27] (a) Gayam, S. R.; Venkatesan, P.; Sung, Y.-M.; Sung, S.-Y.; Hu, S.-H.; Hsu, H.-Y.; Wu, S.-P. Nanoscale 2016, 8, 12307.

(b) Lee, J.; Oh, E.-T.; Yoon, H.; Woo Kim, C.; Han, Y.; Song, J.; Jang, H.; Joo Park, H.; Kim, C. Nanoscale 2017, 9, 6901.

(c) Cheng, Y.-J.; Luo, G.-F.; Zhu, J.-Y.; Xu, X.-D.; Zeng, X.; Cheng, D.-B.; Li, Y.-M.; Wu, Y.; Zhang, X.-Z.; Zhuo, R.-X.; He, F. ACS Appl. Mater. Interfaces 2015, 7, 9078.

[28] Hu, P.; Chen, Y.; Li, J. J.; Liu, Y. Chem.-Asian J. 2016, 11, 505.

[29] Hou, X.-F.; Chen, Y.; Liu, Y. Soft Matter 2015, 11, 2488.

[30] Han, X.; Chen, Y.; Sun, H.-L.; Liu, Y. Asian J. Org. Chem. 2018, 7, 870.

[31] Guan, X.; Chen, Y.; Wu, X.; Li, P.; Liu, Y. Chem. Commun. 2019 , $55,953$.

[32] (a) Zhao, Q.; Lian, Z.; Gao, X.; Yan, Y.; Huang, J. Langmuir 2016, 32, 11973

(b) Mandl, G. A.; Rojas-Gutierrez, P. A.; Capobianco, J. A. Chem. Commun. 2018, 54, 5847. (c) Stricker, L.; Fritz, E.-C.; Peterlechner, M.; Doltsinis, N. L.; Ravoo, B. J. J. Am. Chem. Soc. 2016, 138, 4547.

[33] Zhao, X.; Chen, Y.; Guan, X. R.; Li, P. Y.; Zhou, W. L.; Liu, Y. ChemistrySelect 2019, 4, 13241.

[34] Li, Z.-Y.; Chen, Y.; Wu, H.; Liu, Y. ChemistrySelect 2018, 3, 3203.

[35] Liu, Z.; Qiao, J.; Tian, Y.; Wu, M.; Niu, Z.; Huang, Y. Langmuir 2014, 30, 8938 .

[36] Liu, J.; Xu, L.; Jin, Y.; Qi, C.; Li, Q.; Zhang, Y.; Jiang, X.; Wang, G.; Wang, Z.; Wang, L. ACS Appl. Mater. Interfaces 2016, 8, 14200 .

[37] Chen, X.; Qiu, Y. K.; Owh, C.; Loh, X. J.; Wu, Y. L. Nanoscale 2016, 8, 18876.

[38] Wen, Y.; Zhang, Z.; Li, J. Adv. Funct. Mater. 2014, 24, 3874.

[39] (a) Yang, C.; Wang, X.; Li, H.; Goh, S. H.; Li, J. Biomacromolecules 2007, 8, 3365.

(b) Yamashita, A.; Kanda, D.; Katoono, R.; Yui, N.; Ooya, T.; Maruyama, A.; Akita, H.; Kogure, K.; Harashima, H. J. Control. Release 2008, 131, 137.

[40] Ooya, T.; Choi, H. S.; Yamashita, A.; Yui, N.; Sugaya, Y.; Kano, A.; Maruyama, A.; Akita, H.; Ito, R.; Kogure, K.; Harashima, H. J. Am. Chem. Soc. 2006, 128, 3852.

[41] Tamura, A.; Yui, N. Biomaterials 2013, 34, 2480.

[42] Schroeder, T. B. H.; Houghtaling, J.; Wilts, B. D.; Mayer, M. $A d v$. Mater. 2018, 30, 1705322.

[43] (a) Badruddoza, A. Z. M.; Bhattarai, B.; Suri, R. P. S. ACS Sustainable Chem. Eng. 2017, 5, 9223.

(b) Lu, A.-H.; Salabas, E. L.; Schüth, F. Angew. Chem., Int. Ed. 2007, 46, 1222.

[44] Samanta, A.; Ravoo, B. J. Angew. Chem., Int. Ed. 2014, 53, 12946.

[45] Awasthi, A. A.; Singh, P. K. J. Phys. Chem. B 2017, 121, 6208.

[46] Singh, G.; Singh, P. K. Langmuir 2019, 35, 14628.

[47] Hu, P.; Chen, Y.; Liu, Y. Chem. Commun. 2015, 51, 10839. 